\title{
Effects of hadronic rescattering on multistrange hadrons in high-energy nuclear collisions
}

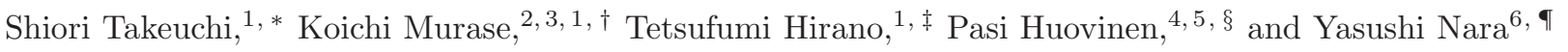 \\ ${ }^{1}$ Department of Physics, Sophia University, Tokyo 102-8554, Japan \\ ${ }^{2}$ Department of Physics, The University of Tokyo, Tokyo 113-0033, Japan \\ ${ }^{3}$ Theoretical Research Division, Nishina Center, RIKEN, Wako 351-0198, Japan \\ ${ }^{4}$ Institut für Theoretische Physik, Goethe-Universität, 60438 Frankfurt am Main, Germany \\ ${ }^{5}$ Frankfurt Institute for Advanced Studies, 60438 Frankfurt am Main, Germany \\ ${ }^{6}$ Department of International Liberal Arts, Akita International University, Yuwa, Akita-city 010-1292, Japan
}

(Dated: August 30, 2018)

\begin{abstract}
We study the effects of hadronic rescattering on hadron distributions in high-energy nuclear collisions by using an integrated dynamical approach. This approach is based on a hybrid model combining (3+1)-dimensional ideal hydrodynamics for the quark gluon plasma (QGP), and a transport model for the hadron resonance gas. Since the hadron distributions are the result of the entire expansion history of the system, understanding the QGP properties requires investigating how rescattering during the hadronic stage affects the final distributions of hadrons. We include multistrange hadrons in our study, and quantify the effects of hadronic rescattering on their mean transverse momenta and elliptic flow. We find that multistrange hadrons scatter less during the hadronic stage than non-strange particles, and thus their distributions reflect the properties of the system in an earlier stage than the distributions of non-strange particles.
\end{abstract}

PACS numbers: 25.75.Ld, 12.38.Mh, 24.10.Nz, 25.75.Dw

\section{INTRODUCTION}

Quark gluon plasma (QGP), strongly interacting matter composed of quarks and gluons, has been created in high-energy nuclear collisions at the Relativistic Heavy Ion Collider (RHIC) at Brookhaven National Laboratory (BNL) and the Large Hadron Collider (LHC) at CERN [1]. One of the main discoveries at the RHIC was that the QGP behaves like a nearly perfect fluid [27], and since then the transport properties of the QGP, especially its shear and bulk viscosity coefficients, have been under active investigation.

The QGP created in high-energy nuclear collisions expands, cools down, and turns into a hadron gas. Since hadrons scatter with each other via the strong interaction, information about the QGP stage is, in general, contaminated by rescatterings in the hadronic stage. To probe the QGP more directly, thermal photons and dileptons were proposed as penetrating probes [8, [9]. Once emitted, photons and dileptons propagate to detectors without rescattering since they interact only electromagnetically and their mean free path is therefore longer than the typical size of the system. However, photons and dileptons are not perfect probes of the QGP either. They are emitted during all the stages of high-energy nuclear collisions such as the primary hard scatterings,

\footnotetext{
*Electronic address: takeuchi@sophia.ac.jp

${ }^{\dagger}$ Electronic address: murase@nt.phys.s.u-tokyo.ac.jp

‡Electronic address: hirano@sophia.ac.jp

$\S$ Electronic address: huovinen@th.physik.uni-frankfurt.de

ฯ Electronic address: nara@aiu.ac.jp
}

pre-equilibrium stage, and late hadronic stage [10], and it is not possible to distinguish photons and dileptons emitted from the QGP from those originating from the other processes.

In this paper, we follow up the previous studies 11 17] about multistrange hadrons, and claim that their distributions allow to probe the QGP immediately after hadronization. Multistrange hadrons, in particular $\phi$ mesons and $\Omega$ baryons, have small scattering crosssections with pions since they do not form any resonances unlike other hadrons. This is a unique property of the multistrange hadrons: Their distributions reflect the properties of the system mainly at a specific stage deep inside the fireball, unlike photons and dileptons which come from the all stages.

By using a hybrid model in which hydrodynamic description of the QGP fluid is followed by a hadronic cascade model, violation of the mass ordering of the differential elliptic flow parameter $v_{2}\left(p_{T}\right)$ was predicted [15]. This phenomenon was observed recently by the STAR Collaboration [18], which indicates that $\phi$ mesons are less affected by hadronic rescatterings and, consequently, that they are good probes deep inside the matter. In this paper, we investigate the hadronic rescattering effects on observables more systematically by employing an integrated dynamical approach which we have previously used to analyze various observables at the RHIC and the LHC energies 19]. In the previous study of the violation of mass ordering [15], a first order phase transition model for the equation of state (EoS) was employed and only hadrons up to the mass of $\Delta(1232)$ were taken into account in the hadron phase. In the present paper, we use a more realistic equation of state [20] connecting a parametrized lattice QCD EoS at large temperatures 
to a hadron resonance gas EoS at low temperatures. The hadron resonance gas in the EoS contains all the resonances in the hadron cascade model JAM (Jet $A A$ Microscopic transport model) 21] used as the last stage of the integrated dynamical approach. Hence, in addition to $\phi$, we are able to investigate hadronic rescattering effects on heavier multistrange hadrons such as $\Xi$ and $\Omega$.

In the following we introduce the integrated dynamical model in Sec. II We compare transverse momentum $\left(p_{T}\right)$ spectra and $p_{T}$-differential elliptic flow parameters $v_{2}\left(p_{T}\right)$ with the STAR data in Sec. III] After we confirm we reasonably reproduce the data by using this model, we discuss the hadronic rescattering effect in Sec. IV] In this section, we first revisit the violation of mass ordering of $v_{2}\left(p_{T}\right)$. We next focus on the mean transverse momentum $\left\langle p_{T}\right\rangle$ and $p_{T}$-averaged $v_{2}$ calculated with or without hadronic rescatterings and investigate how much these final observables reflect the information when the hydrodynamic stage finishes. We summarize our results in Sec. V]

\section{THE MODEL}

We describe the space-time evolution of high-energy nuclear collisions by an integrated dynamical approach on an event-by-event basis. We divide the whole reaction into three separate stages: Initial stage, hydrodynamic stage, and transport stage. The initial entropy density distribution after the collision of energetic heavy nuclei is parametrized by using a Monte-Carlo version of the Glauber model. The subsequent expansion of the matter is described by relativistic ideal hydrodynamics. When the system becomes sufficiently dilute due to strong expansion, we switch the description of the system from hydrodynamics to kinetic theory.

In the following we briefly overview each part of the model and the corresponding interfaces. For further details, see Ref. [19].

\section{A. Hydrodynamics and equation of state}

Hydrodynamics is a macroscopic description and an effective theory of the system's long wavelength/time behavior. The hydrodynamical equations of motion are obtained from the conservation of energy and momentum:

$$
\partial_{\mu} T^{\mu \nu}=0,
$$

where $T^{\mu \nu}$ is energy-momentum tensor. In the ideal fluid approximation, the energy momentum tensor can be decomposed as

$$
T^{\mu \nu}=e u^{\mu} u^{\nu}-P\left(g^{\mu \nu}-u^{\mu} u^{\nu}\right),
$$

where $e, P, u^{\mu}$, and $g^{\mu \nu}=\operatorname{diag}(+1,-1,-1,-1)$ are energy density, pressure, flow velocity, and the Minkowski metric, respectively. To close the system of partial differential equations we need to know the equation of state (EoS) of the fluid to obtain pressure $P$ as a function of energy density $e$. Once the EoS is known, the space-time evolution of thermodynamic quantities and flow velocity is determined for given initial conditions, i.e., energy density distribution and flow field at initial time $\tau_{0}$.

We ignore the baryon number and its continuity equation since at the collider energies, net baryon density at midrapidity is tiny. When we solve Eq. (10), temperature is not needed since it is merely a parameter to connect energy density and pressure. Nevertheless, we also follow the space-time evolution of temperature to decide where to switch from fluid to cascade. We employ the $s 95 p$ v1.1 parametrization for the EoS. The $s 95 p$ parametrization [20] connects the lattice QCD based EoS [22] in the high temperature region to a hadron resonance gas EoS in the low temperature region. The hadronic part of $s 95 p$-v1.1 contains the same hadronic species as the JAM hadronic cascade model [21] described in the next subsection.

We numerically solve Eq. (11) in the Milne coordinates $\left(\tau, \eta_{s}, x, y\right)$ which is appropriate for the description of the evolution at relativistic energies 23. Here $\tau=\sqrt{t^{2}-z^{2}}$ is longitudinal proper time, $\eta_{s}=\frac{1}{2} \ln [(t+z) /(t-z)]$ is space-time rapidity, and $x$ and $y$ are transverse coordinates perpendicular to the collision axis. In the numerical calculations, we employ the Piecewise Parabolic Method [24] which is known to be able to describe shock waves. For details about this numerical algorithm, see also Ref. [25].

\section{B. Particlization and hadronic cascade}

We employ the hadronic cascade model JAM [21] to describe the space-time evolution of hadron gas after "particlization", i.e., after switching from fluid to particles. At the late stage of collisions, the system is too dilute to maintain equilibrium. We assume this happens around temperature, which we call the switching temperature $T_{\mathrm{sw}}$, and switch description from hydrodynamics to kinetic theory on $T=T_{\mathrm{sw}}$ isosurface 13 , 26 31]. Within the current model, $T_{\mathrm{sw}}$ is rather adjustable parameter which controls the final particle ratios. We choose $T_{\mathrm{sw}}=155 \mathrm{MeV}$ to reproduce the observed $p_{T}$ spectra of pions, kaons, and protons + anti-protons in the low $p_{T}$ region [19] at the full RHIC energy. At $T(x)=T_{\mathrm{sw}}$, we calculate the single particle phase space distributions $f_{i}(p, x)$ for all hadrons included in the EoS by employing the Cooper-Frye prescription [32]

$$
f_{i}(p, x) d^{3} \boldsymbol{x} d^{3} \boldsymbol{p}=\frac{g_{i}}{(2 \pi)^{3} E} \frac{p \cdot \Delta \sigma d^{3} \boldsymbol{p}}{\exp \left(p \cdot u / T_{\mathrm{sw}}\right) \pm 1} .
$$

Here $g_{i}$ is a degeneracy of the hadronic species $i, p^{\mu}=$ $(E, \boldsymbol{p})$ is four-momentum of a particle, and $\Delta \sigma_{\mu}$ is a normal vector of the $T(x)=T_{\mathrm{sw}}$ hypersurface. It is well 
known that at some momenta the Cooper-Frye formula (3) gives negative contribution for space-like hypersurface element (or even time-like hypersurface element with negative time component). These negative numbers correspond to in-coming particles, which one cannot treat in the hadronic cascade model. So we just neglect these contributions and consider only out-going particles. We sample particles by going through all the hypersurface elements and generate ensembles of hadrons on an eventby-event basis. Note that we do not oversample particles to gain statistics for each event. This means each hydrodynamic event corresponds to one ensemble of particles. Among all the calculations in the integrated dynamical approach, sampling particles from particlization hypersurface is numerically most expensive, which is crucial in event-by-event simulations. In Ref. [19], we discussed in detail how to sample particles and integrate the CooperFrye formula (3) over momentum at less numerical cost.

In hadronic cascade models, experimental hadronic cross section data are implemented when available. However, there are many hadronic scattering processes where data do not exist. In such a case, we use the additive quark model [33 36] to describe the scattering cross section

$$
\sigma_{\text {tot }}^{12}=\sigma_{\text {tot }}^{N N} \frac{n_{1}}{3} \frac{n_{2}}{3}\left(1-0.4 \frac{n_{s 1}}{n_{1}}\right)\left(1-0.4 \frac{n_{s 2}}{n_{2}}\right) .
$$

Here $\sigma_{\text {tot }}^{N N}$ is the total nucleon-nucleon cross section, $n_{i}$ is the number of constituent quarks in a hadron, and $n_{s i}$ is the number of strange quarks in a hadron. Thus hadrons containing strange quark(s) have relatively small total cross section in this model. Moreover, $\phi$ mesons and $\Omega$ baryons do not form resonances when scattering with pions and therefore have much smaller cross sections than non-strange hadrons.

In the simulations, dynamics of the system is described by a sum of incoherent two-body collisions. Two particles collide with each other if their minimum distance $b$ in the center of mass frame is smaller than the distance given by the total cross section

$$
b<\sqrt{\frac{\sigma_{\mathrm{tot}}}{\pi}} .
$$

This is a classical, geometrical interpretation of the twobody collision cross section.

Note that in JAM the properties of hadrons are those of free particles. The medium modifications of hadron properties such as a broadening and mass shift 37. have been extensively studied by various further developments of transport models such as the relativistic VlasovUehling-Uhlenbeck (RVUU) model [38], the hadronstring-dynamics (HSD [39]) and parton-hadron-stringdynamics (PHSD [40]) transport models, the Giessen Boltzmann-Uehling-Uhlenbeck (GiBUU) 41], and the Isospin Quantum Molecular Dynamics (IQMD) [42]. Nevertheless, since the main difference in the cross sections of multistrange particles and the other hadrons is due to the (non-)existence of resonance channels, we do not expect the in-medium modifications to significantly affect this difference, and the use of free particle properties to provide a conservative baseline is justified.

As mentioned, the interactions in JAM are described as two particle scatterings, and multi-particle scatterings are not included. It has been argued that multi-pion fusion processes in particular would have a significant effect on the yields of proton-antiproton pairs [43], but subsequent calculations have shown that regeneration is $\mathrm{a} \sim 10 \%$ level effect at the LHC [44, 45]. Nevertheless, the multi-meson fusion effects for $\Omega$ baryons are much smaller than for protons or $\Lambda$ [46], and thus their inclusion would make the later-discussed differences between rescatterings of non-strange and multistrange particles even larger.

As a default setting strong interactions and decays are simulated in JAM. However, we switch off the decay channels for $\phi$ mesons in the present study to investigate hadronic rescattering effects on $\phi$ mesons in an efficient way. Otherwise, we would need to perform mass reconstruction of $\phi$ mesons from two kaons after numerical simulations. Since the lifetime of $\phi$ mesons $(\sim 47 \mathrm{fm} / c)$ [47] is somewhat larger than the typical life time of the system $(\sim 10 \mathrm{fm} / c)$, the daughter kaons from $\phi$ meson decays (or lack of them) are not expected to affect the bulk evolution. As we will discuss in Sec. IIIT, the yield of $\Lambda$ measured by STAR contains the feed down from electromagnetic $\Sigma^{0}$ decays. This process does not happen in the default setting in JAM and, therefore, is simulated separately when we analyze $\Lambda$ spectra. We also have an option in JAM to deactivate all the hadronic rescatterings and/or resonance decays to investigate how these affect final observables.

\section{Initial conditions}

Once initial conditions for the hydrodynamic evolution are fixed, the subsequent evolution is determined, and we are able to obtain final particle distributions which can be compared with experimental data. The pre-thermalization stage between the first contact of colliding nuclei and the initial time of hydrodynamic evolution may be described by non-equilibrium field theory, although how exactly it could be done is one of the open issues in the physics of relativistic heavy ion collisions. Thus, we do not even try to describe it and simply rely on the Monte-Carlo (MC) Glauber [48] and the modified Brodsky-Gunion-Kuhn (BGK) models [29] to give the density distribution at $\tau_{0}$ in the transverse plane and in the longitudinal direction, respectively. The MC Glauber model enables us to calculate the number density of participants and of binary collisions on an event-by-event basis. Our assumption is that the initial entropy density is proportional to a linear combination of these two densities. Even at very high collision energies matter profile in the longitudinal direction depends on space-time ra- 
pidity, and boost invariance does not hold. Furthermore, in non-central collisions the longitudinal density gradient at mid space-time rapidity may be non-zero due to the different thicknesses of the colliding nuclei at each transverse position. This can be deduced from (pseudo)rapidity distribution of hadrons in $\mathrm{p}(\mathrm{d})$-A collisions. In the modified BGK model smooth initial longitudinal profile is parametrized by taking account of the difference in the local thickness of colliding nuclei. It is noted that we do not employ the Monte-Carlo Kharzeev-Levin-Nardi model [49, 50] which was used to calculate initial conditions in our previous hydrodynamic studies [19] since, in the present study, we focus on the hadronic rescattering effects on transverse dynamics, which are not supposed to be sensitive to the choice of initial conditions.

Assuming local thermalization, energy density distribution $e\left(\tau=\tau_{0}, \eta_{s}, x, y\right)$ and pressure $P\left(\tau=\tau_{0}, \eta_{s}, x, y\right)$ are obtained from the entropy density distribution through the $s 95 p$-v1.1 EoS. Initial flow velocity at $\tau_{0}$ is supposed to be Bjorken's scaling flow [51], namely, $u^{\tau}=1$ and $u^{x}=u^{y}=u^{\eta_{s}}=0$. Throughout this study, we fix $\tau_{0}=0.6 \mathrm{fm} / c$. We choose initial parameters to reproduce the $p_{T}$ distributions of pions, kaons, and protons + antiprotons in $\mathrm{Au}+\mathrm{Au}$ collisions at $\sqrt{s_{N N}}=200 \mathrm{GeV}$ measured by the PHENIX Collaboration [52]. The centrality dependence of multiplicity is controlled by a fraction of soft (participants) and hard (binary collisions) component in the MC Glauber model, whereas the multiplicity in central collisions is fixed by the overall normalization parameter of the initial entropy distribution. On the other hand, as mentioned particle ratios are controlled by the switching temperature. The parameters controlling the shape of the rapidity distribution of particles are kept the same as in our previous study [19]. Since here we concentrate on observables at midrapidity, we do not discuss them further.

In hydrodynamic simulations, one needs to convert the position distribution (a sum of delta functions in the transverse plane) of collision points in MC Glauber model to a hydrodynamic density distribution. In our model we obtain the density at a point $x$ by counting the number of collision points or participants within radius $r_{0}=\sqrt{\sigma_{\mathrm{in}} / \pi}$ around the point, and divide by the inelastic cross section in $p+p$ collisions at $\sqrt{s_{N N}}=200 \mathrm{GeV}$, $\sigma_{\text {in }}$. For further details, see Ref. [19].

\section{SPECTRA AND FLOW}

In this section, we compare invariant $p_{T}$ spectra and differential elliptic flow parameter $v_{2}\left(p_{T}\right)$ of identified hadrons with experimental data from the STAR Collaboration. In the present study, we analyzed 0.6 million "minimum bias" events (defined as $N_{\text {part }} \geq 2$ in the MC Glauber calculations for hydrodynamic initial conditions). Centrality is defined using an event distribution of the charged hadron multiplicity in $|\eta|<0.5$ as done by the STAR Collaboration [53]. Note that error bars in the theoretical plots denote the statistical errors.

\section{A. Transverse momentum spectra}

In Figs. 1, 2, and 3, we show $p_{T}$ distributions of identified hadrons around midrapidity in $\mathrm{Au}+\mathrm{Au}$ collisions at $\sqrt{s_{N N}}=200 \mathrm{GeV}$. For clarity, the results and the experimental data points are scaled up or down by a common factor.

Figure 1 shows the $p_{T}$ spectra of (a) positive pions $(|\eta|<0.5)$ and (b) positive kaons $(|\eta|<0.1)$ compared with the STAR data $(|y|<0.5$ for pions [54] and $|y|<0.1$ for kaons [55). The Jacobian between $\eta$ and $y$ is taken into account to obtain the invariant $p_{T}$ spectra in our results. Even if we tuned the initial conditions and the switching temperature in the model using pion, kaon, and proton + anti-proton $p_{T}$ spectra from the PHENIX Collaboration in our previous study, the results are overall in good agreement with the STAR data too.

In particular, the model reproduces the pion data up to $p_{T} \sim 3 \mathrm{GeV} / c$ in central $(0-12 \%)$ collisions. Above this, our result gradually deviates from the data due to appearance of (semi-)hard components such as recombination and jet fragmentation. The more peripheral the collision, the lower the $p_{T}$ where our result begins to deviate from the data. Due to the limited $p_{T}$ range of the data, the same behavior is not seen in the kaon spectra. If one looks at the pion spectrum in peripheral collisions (60-80\% centrality) carefully, one sees that experimental data are systematically larger than the results. This is because in peripheral collisions there is a small discrepancy between PHENIX [52] and STAR [54] pion yields. Nevertheless we did not further tune the parameters to reproduce the STAR data because we want to keep the same framework as in our previous study [19].

In Fig. 2, we compare the $p_{T}$ spectra in $|\eta|<0.5$ for (a) protons and (b) $\phi$ mesons with the STAR data [54, 56]. Due to limited statistics, we do not show some data points of the proton spectra in high $p_{T}$ regions (above $\left.p_{T} \sim 4 \mathrm{GeV} / c\right)$ in $20-40,40-60$, and $60-80 \%$ centrality. The proton spectra from STAR are corrected for the $\Lambda$ and $\Sigma^{+}$feed down. Weak decays do not occur in the default setting in JAM, so we are able to compare our results directly with the STAR corrected data. Since we neglect baryon chemical potential in our model, our results for protons are slightly below the experimental data at some $p_{T}$ range. Nevertheless, overall slopes are in good agreement with the data below $p_{T} \sim 3 \mathrm{GeV} / c$. On the other hand, similar to pions, deviation between the experimental data and the results gradually increases with $p_{T}$, and the more peripheral the collision, the lower the $p_{T}$ where this deviation appears. As mentioned in the previous section, we switch off the decays of $\phi$ mesons in the hadronic cascade to be able to analyze $\phi$ meson spectra directly without resorting to mass reconstruction from kaons. Tendencies seen in $\phi$ meson spectra are similar to those in proton spectra. 

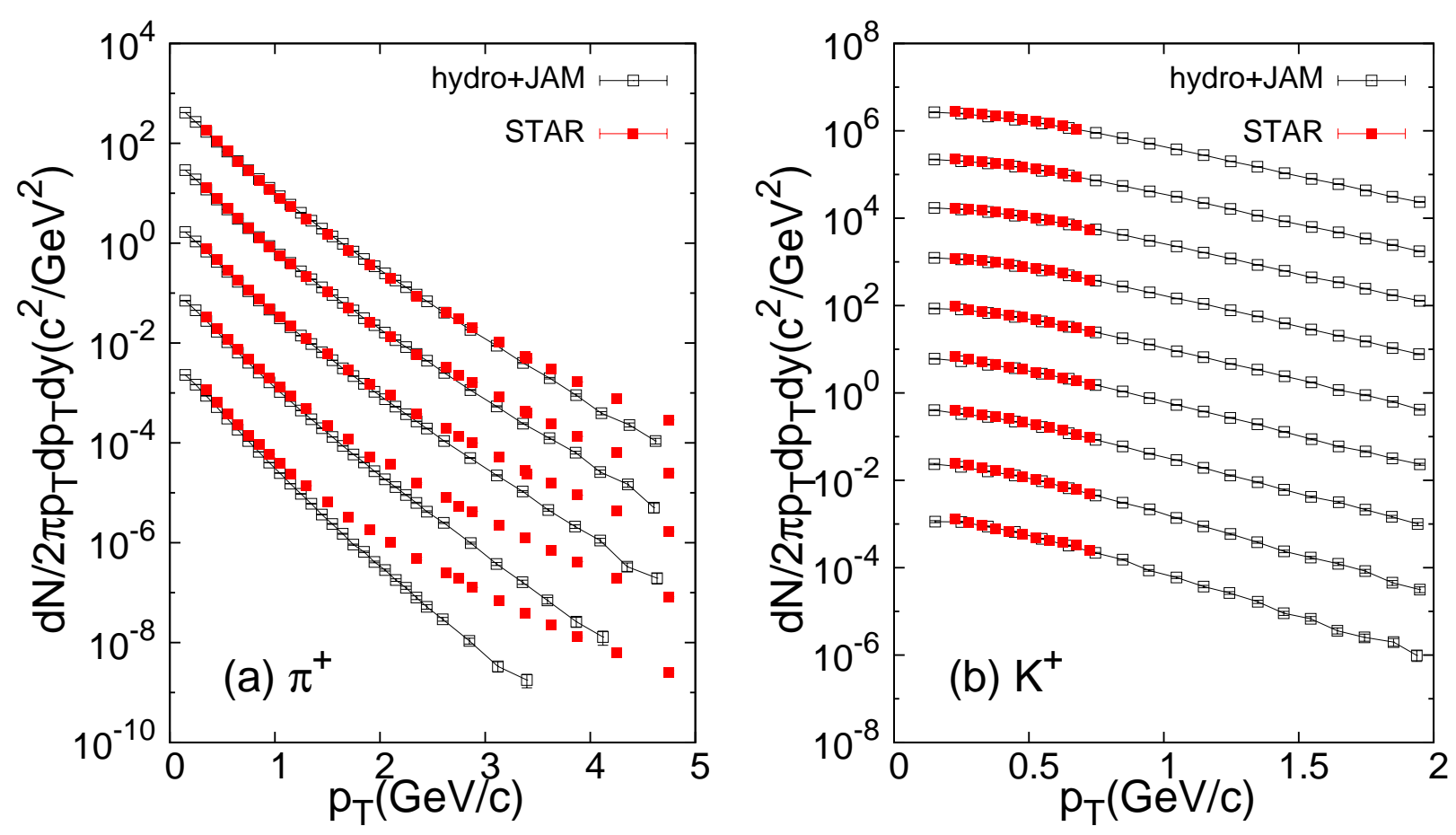

FIG. 1: (Color Online) Transverse momentum distributions of (a) $\pi^{+}(|\eta|<0.5)$ and (b) $K^{+}(|\eta|<0.1)$ obtained from the integrated dynamical approach (open square) compared with data from the STAR Collaboration [54, 55] (filled square) for $\sqrt{s_{N N}}=200 \mathrm{GeV} \mathrm{Au}+\mathrm{Au}$ collisions. From top to bottom, each spectrum shows the result of 0-12, 10-20, 20-40, 40-60, and $60-80 \%$ centrality multiplied by $10^{n}$ with $n=0$ to -4 for pions, and $0-5,5-10,10-20,20-30,30-40,40-50,50-60,60-70$, and $70-80 \%$ centrality multiplied by $10^{n}$ with $n=5$ to -3 for kaons.

Figure 3 shows the $p_{T}$ spectra of strange baryons, (a) $\Lambda+\bar{\Lambda}(|\eta|<1.0)$, (b) $\Xi+\bar{\Xi}(|\eta|<0.75)$, and (c) $\Omega+\bar{\Omega}(|\eta|<0.75)$, compared with the STAR data $[57$ ]. Similar to the proton case, the more peripheral the collisions, the worse the agreement between experimental data and the results, especially above $p_{T}=1.5-2.0$ $\mathrm{GeV} / c$. As expected the measured $\Lambda$ and $\Xi$ yields are larger than the calculated yields. We had chosen the switching temperature $T_{\mathrm{sw}}$ to reproduce the observed pion to kaon and pion to proton ratios, which leads to a lower temperature than the statistical model fit to all observed hadrons [58, 59], and thus to lower yields of hyperons. On the other hand it has been argued that the statistical model [60] with the common chemical freezeout temperature does not reproduce the proton and $\Lambda$ yields simultaneously at the LHC and RHIC energies [61]. It was claimed 44] that earlier switching from hydrodynamics to UrQMD at energy density $\sim 840 \mathrm{MeV} / \mathrm{fm}^{3}$, which corresponds to higher temperature than $T_{\mathrm{sw}}=155 \mathrm{MeV}$, would be a key to resolve the issue, but our results do not improve significantly if we use higher switching temperature $T_{\mathrm{sw}}=165 \mathrm{MeV}$. It remains to be seen whether the origin of this discrepancy lies in a different description of the proton-antiproton annihilations in JAM and UrQMD cascades, or in different expansion dynamics in collisions at RHIC and LHC energies.

Note that the experimental yield of $\Lambda$ in STAR con- tains the feed down from electromagnetic $\Sigma^{0}$ decays $\left(\Sigma^{0} \rightarrow \Lambda+\gamma\right)$ 62]. Since this is the dominant mode of $\Sigma^{0}$ decay with the branching ratio $\sim 100 \%$ and $c \tau=$ $2.22 \times 10^{-11} \mathrm{~m}$ [47], the feed down correction of it is experimentally challenging. We simulate this decay in JAM and include this contribution in the final $\Lambda$ spectra in Fig. 3 (a). Since the mass of $\Sigma^{0}$ is close to that of $\Lambda$, the yield of the primordial $\Sigma^{0}$ is expected to be of the same order as the yield of the primordial $\Lambda$. The ratio of $\Sigma^{0}$ to $\Lambda$ at switching temperature can be estimated as

$$
\frac{n_{\Sigma^{0}}}{n_{\Lambda}} \approx \frac{\exp \left(-\frac{m_{\Sigma^{0}}}{T_{\mathrm{sw}}}\right)}{\exp \left(-\frac{m_{\Lambda}}{T_{\mathrm{sw}}}\right)} \sim 0.61 .
$$

After correcting for the effects of resonance decay and rescatterings, we find that the final particle ratio of $\Sigma^{0}$ to $\Lambda$ is around 0.3. We have included both sources of $\Lambda$ in our results, but in general this should be kept in mind when comparing theoretical results with the data.

Although the number of data points from STAR is limited for $\Omega$ baryons, their yields and slopes are consistent with our results. It is worth noticing here that the recent lattice QCD calculations suggest the existence of reso- 

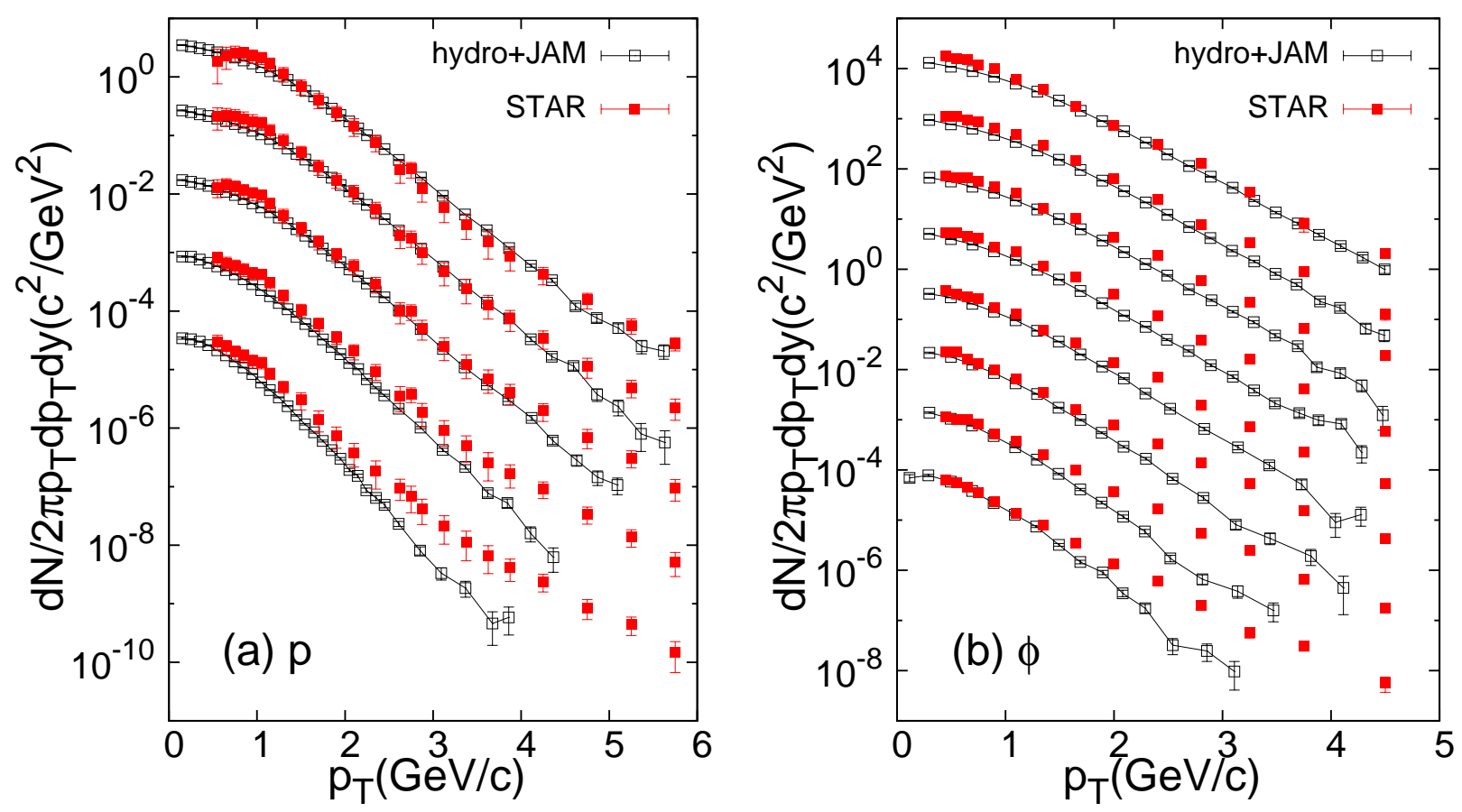

FIG. 2: (Color Online) Transverse momentum distributions of (a) protons and (b) $\phi$ mesons in $|\eta|<0.5$ obtained from the integrated dynamical approach (open square) compared with data from the STAR Collaboration [54, 56] (filled square) for $\sqrt{s_{N N}}=200 \mathrm{GeV} \mathrm{Au}+\mathrm{Au}$ collisions. From top to bottom, each spectrum shows the result of 0-12, 10-20, 20-40, 40-60, and $60-80 \%$ centrality multiplied by $10^{n}$ with $n=0$ to -4 for protons, and $0-10,10-20,20-30,30-40,40-50,50-60,60-70$, and $70-80 \%$ centrality multiplied by $10^{n}$ with $n=4$ to -3 for $\phi$ mesons.

nances in general $[63]^{1}$, or strange baryon resonances in particular [22], which have not been discovered yet.

Such resonances, whether they are of Hagedorn kind [65], or those predicted by quark models (see e.g. Refs. 66, 67]), would contribute to the yields of strange hadrons, and might somewhat change the dynamics of the late hadronic stage 68 70]. Some of those resonances could be formed in the scattering of $\phi$ mesons or $\Omega$ baryons, but it is unknown whether such resonances would significantly affect the average scattering cross section of $\phi$ mesons and $\Omega$ baryons in temperatures below the switching temperature. Investigation of this possibility is beyond the scope of the present paper.

\section{B. Elliptic flow}

In Fig. 4 the two-particle cumulant $v_{2}\left(p_{T}\right)$ of pions, kaons, and protons in the midrapidity region $(|\eta|<1.0)$ are compared with STAR $v_{2}\{2\}$ data 71 ] at three centralities $(10-20 \%, 30-40 \%$, and $50-60 \%)$. For pions, we

\footnotetext{
${ }^{1}$ It is, however, unknown whether the difference between the lattice QCD and hadron resonance gas model trace anomalies indicates the inadequacy of the hadron resonance gas model [64], or the existence of so far undiscovered resonance states 63 .
}

reasonably reproduce the experimental data for each centrality class. Due to the limited number of events, it is hard to obtain $v_{2}\{2\}$ with smaller statistical errors, and especially difficult this would be for protons. Thus some points with large error bars, in particular in low $p_{T}$ region, are not shown in these figures for clarity. We also calculated $v_{2}\{\mathrm{RP}\}$ (not shown), namely $v_{2}$ with respect to theoretically-known reaction plane, to reduce the statistical errors and found that $v_{2}\{\mathrm{RP}\}$ of pions, kaons, and protons from our model are in reasonable agreement with the STAR data in mid-central collisions. However, $v_{2}\{\mathrm{RP}\}$ lacks initial fluctuations of the event plane angle and, consequently, is significantly smaller than the data in both central and peripheral collisions. It is noted that, from the MC Glauber model analysis, the fluctuation effect on eccentricity is large in central and peripheral collisions, but almost negligible in mid-central collisions. See, e.g., Fig. 3 (a) in Ref. 72]. At the centralities shown in Fig. 团a clear mass ordering behavior among pions, kaons, and protons, namely larger $v_{2}$ for smaller mass at fixed $p_{T}$, is seen both in our results and in the STAR data.

In Fig. 5 we show the event plane $v_{2}\left(p_{T}\right)$ of $\phi, \Lambda$, $\Xi$, and $\Omega$ at some centrality classes from the integrated dynamical approach. In comparison, STAR data from the event plane method $v_{2}\{\mathrm{EP}\}[56,73]$ are also shown in the figure. We calculate $v_{2}\{\mathrm{EP}\}$ by employing the $\eta$ subevent method using hadrons in $|\eta|<1.0$ as done by 

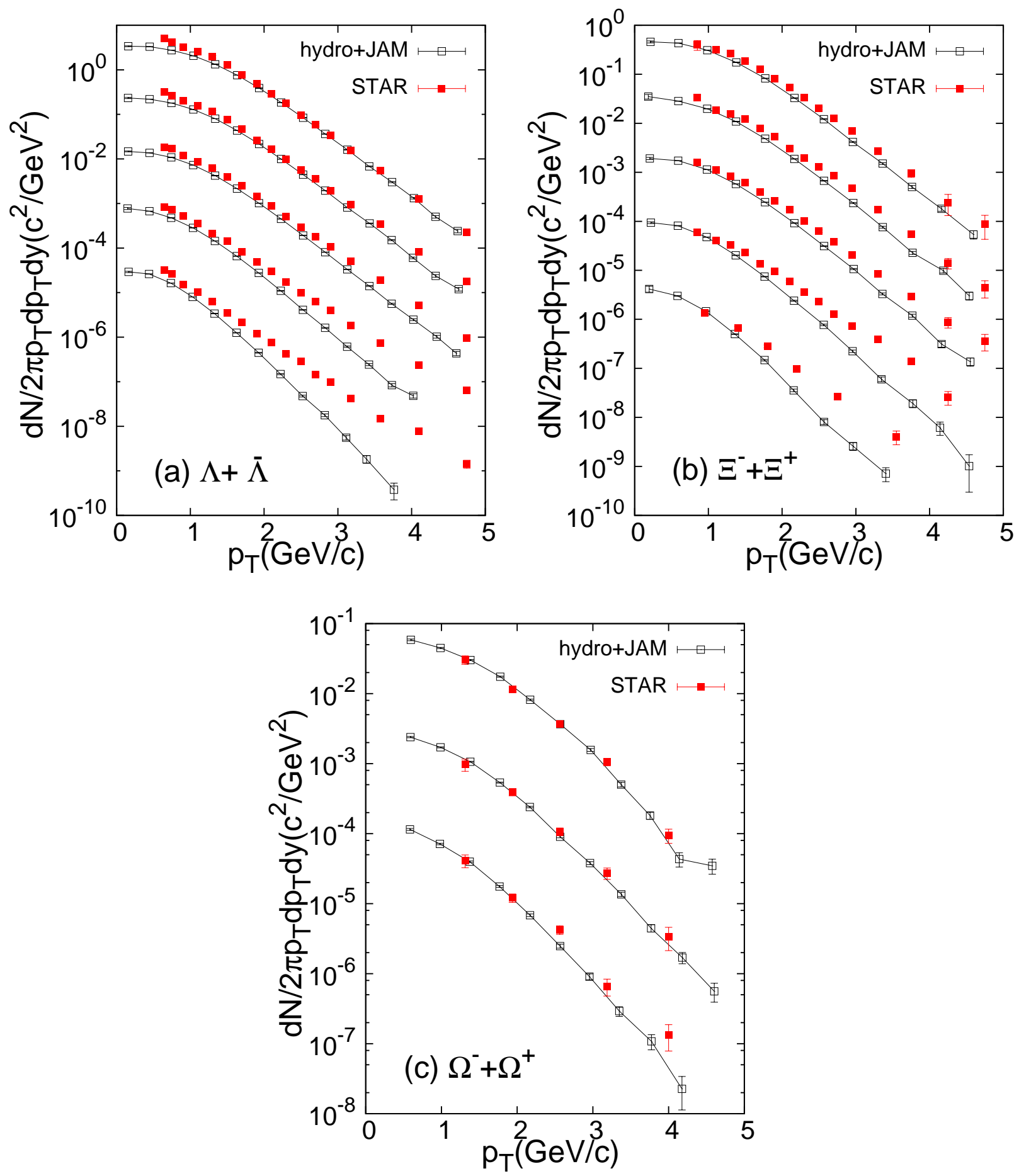

FIG. 3: (Color Online) Transverse momentum distributions of (a) $\Lambda$ ( $|\eta|<1.0)$, (b) $\Xi(|\eta|<0.75)$, and (c) $\Omega(|\eta|<0.75)$ obtained from the integrated dynamical approach (open square) compared with data from the STAR Collaboration [57] (filled square) for $\sqrt{s_{N N}}=200 \mathrm{GeV} \mathrm{Au}+\mathrm{Au}$ collisions. From top to bottom, each spectrum shows data of 0-5, 10-20, 20-40, 40-60, and $60-80 \%$ centrality multiplied by $10^{n}$ with $n=0$ to -4 for $\Lambda$ and $\Xi$ and $0-5,20-40$, and $40-60 \%$ centrality multiplied by $10^{n}$ with $n=0$ to -2 for $\Omega$. Note that the $p_{T}$ distributions of $\Lambda$ include the contribution from $\Sigma^{0}$ decay, see the text for details. 

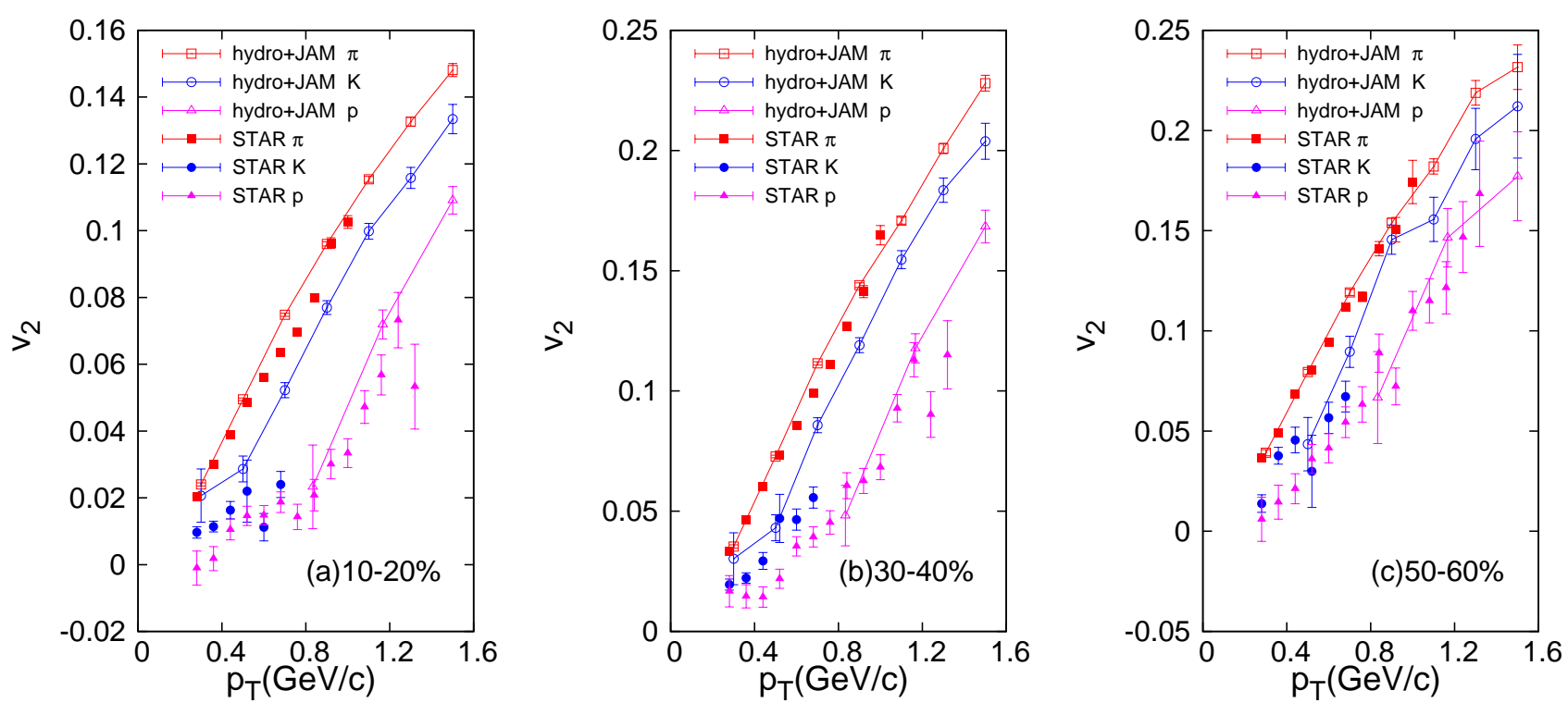

FIG. 4: (Color Online) Transverse momentum dependence of elliptic flow parameter $v_{2}\{2\}$ of pions (open square), kaons (open circle), and protons (open triangle) in $|\eta|<1.0$ obtained from the integrated dynamical approach compared with $v_{2}\{2\}$ data from the STAR Collaboration [71] (filled symbol) for $\sqrt{s_{N N}}=200 \mathrm{GeV} \mathrm{Au}+\mathrm{Au}$ collisions. Centrality classes are (a) 10-20\%, (b) $30-40 \%$, and (c) $50-60 \%$.

the STAR Collaboration [7]],

$$
v_{2}\{\mathrm{EP}\}=\frac{1}{\mathcal{R}}\left\langle\cos \left[2\left(\phi_{ \pm}-\Psi_{2, \mp}\right)\right]\right\rangle .
$$

The event plane angle $\Psi_{2,+}\left(\Psi_{2,-}\right)$ is defined for the particles with positive (negative) pseudorapidity. In the actual experimental analysis, an pseudorapidity gap of $|\Delta \eta|=0.075$ between two subevents was introduced to suppress the nonflow effects 73]. We found, however, that introduction of the gap does not change final results within error bars in our analysis. The resolution factor in this case is calculated as $\mathcal{R}=\sqrt{\left\langle\cos \left[2\left(\Psi_{2,+}-\Psi_{2,-}\right)\right]\right\rangle}$. In calculating the $Q$ vector,

$$
Q=\sum_{j} w_{j} \exp \left(2 i \phi_{j}\right)
$$

needed for the event plane angle

$$
\Psi_{2, \pm}=\frac{1}{2} \tan ^{-1} \frac{\Im Q}{\Re Q},
$$

a weight factor $w_{j}=\min \left\{p_{T, j}, 2 \mathrm{GeV} / c\right\}$ was also introduced [73]. However, within statistical errors the results are similar to those obtained using $w_{j}=1$. Therefore, we employ $w_{j}=1$ in all the results shown in Fig. [5 For $\Lambda$ and $\Xi$ our results are systematically larger than the STAR data. For $\phi$ and $\Omega$ our results show similar behavior, but since the data have large errors, no firm conclusion can be made.

Our results of elliptic flow parameters for identified hadrons are systematically larger than the STAR data, which suggests that there is room for finite, but perhaps small, viscosity in the fluid-dynamical stage. However, we do not go into details about the QGP viscosity since the purpose of the present study is to investigate the effects of rescattering in the late hadronic stage, which are not affected by the QGP viscosity nor by the initial state of the system.

\section{HADRONIC RESCATTERING EFFECTS}

In this section, we investigate hadronic rescattering effects on final observables, in particular, how much final observables reflect the properties of the system at particlization in the integrated dynamical approach. If some observables do not change much during the late kinetic stage, these observables can be utilized as "penetrating" probes. Namely, their distributions reflect the properties of the system immediately after hadronization, and are not contaminated by later hadronic rescatterings. To quantify the effects of hadronic rescattering we perform simulations of nuclear collisions with the following three options in the hadronic cascade calculations: (I) full dynamical evolution (default), (II) deactivating hadronic rescatterings, and (III) deactivating both hadronic rescatterings and resonance decays. To simplify the calculations we calculate elliptic flow parameters with respect to the theoretically-known reaction plane.

In Figs. 6 (a) and (b), we show $v_{2}\left(p_{T}\right)$ of pions, kaons, protons, and $\phi$ mesons in minimum bias $\mathrm{Au}+\mathrm{Au}$ colli- 

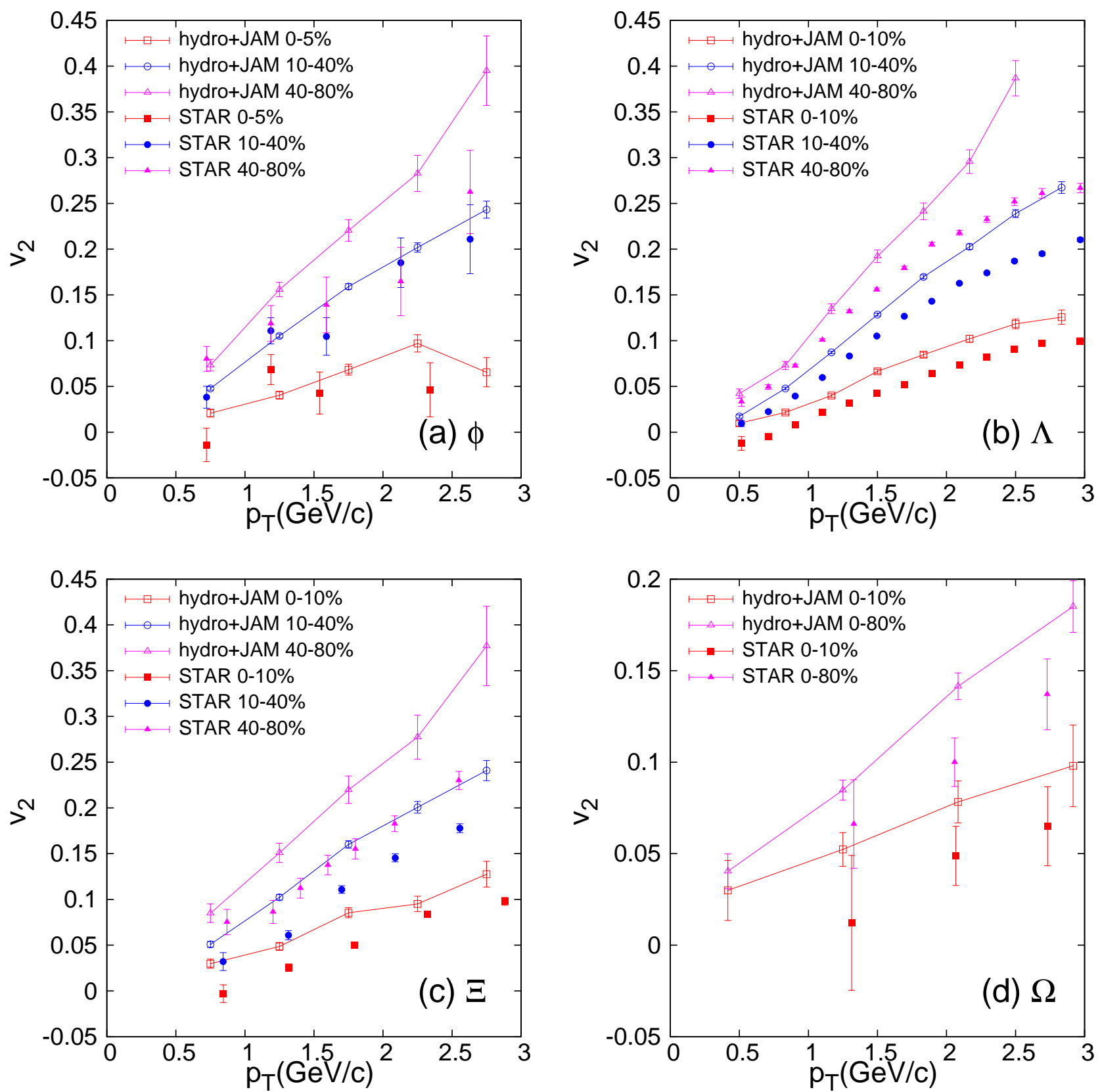

FIG. 5: (Color Online) Transverse momentum dependence of the elliptic flow parameter $v_{2}\{\mathrm{EP}\}$ of (a) $\phi$ mesons $(0-5,10-40$, and $40-80 \%$ centrality), (b) $\Lambda$ baryons (0-10, 10-40, and 40-80\% centrality), (c) $\Xi$ baryons (0-10, 10-40, and 40-80\% centrality), and (d) $\Omega$ baryons (0-10 and 0-80\% centrality) in $|\eta|<1.0$ obtained from the integrated dynamical approach (open symbol) compared with data from the STAR Collaboration [56, 73] (filled symbol) for $\sqrt{s_{N N}}=200 \mathrm{GeV} \mathrm{Au}+\mathrm{Au}$ collisions.

sions at $\sqrt{s_{N N}}=200 \mathrm{GeV}$ from the integrated dynamical approach. Figure 6 (a) represents the results without hadronic rescatterings (option (II)). These results exhibit the mass ordering behavior, namely $v_{2}^{\pi}\left(p_{T}\right)>v_{2}^{K}\left(p_{T}\right)>$ $v_{2}^{p}\left(p_{T}\right)>v_{2}^{\phi}\left(p_{T}\right)$ for $m_{\pi}<m_{K}<m_{p}<m_{\phi}$, in the low $p_{T}$ region. In general the mass ordering results from collective flow in which all the components of the fluid flow at a common fluid velocity 74 . On the other hand,
Fig. 6 (b) shows a violation of mass ordering between protons and $\phi$ mesons below $p_{T} \sim 1.5 \mathrm{GeV} / c: v_{2}^{p}<v_{2}^{\phi}$ even though $m_{p}<m_{\phi}$. If one compares these two plots, $v_{2}^{p}\left(p_{T}\right)$ decreases below $\sim 1.5 \mathrm{GeV} / c$ during the hadronic rescattering stage. On the other hand, $v_{2}^{\phi}\left(p_{T}\right)$ stays almost unchanged during the hadronic rescattering stage. As a consequence, the order is inversed between protons and $\phi$ mesons. To see this behavior more clearly, we also 
show the ratio $v_{2}^{\phi} / v_{2}^{p}$. Below $1 \mathrm{GeV} / c$, this ratio becomes clearly positive indicating the violation of mass ordering.

The violation of mass ordering was predicted in Ref. 15 and observed recently by the STAR Collaboration [18]. It should be noted that a larger switching temperature $\left(T_{\mathrm{sw}}=169 \mathrm{MeV}\right)$ and a first order phase transition in the EoS were employed in the calculations of Ref. [15]. We confirm in the present study that the violation of mass ordering is also observed when a more realistic EoS from lattice QCD and a lower switching temperature $\left(T_{\mathrm{sw}}=155 \mathrm{MeV}\right)$ are used.

We can interpret the violation of mass ordering as a result of evolution of mean $p_{T}$ and $p_{T}$-averaged $v_{2}$ during the late transport stage. The slope of the $p_{T}$-differential $v_{2}$ can be approximated by the ratio of $p_{T}$-averaged $v_{2}$ to mean $p_{T}$ [7],

$$
\frac{d v_{2}\left(p_{T}\right)}{d p_{T}} \approx \frac{v_{2}}{\left\langle p_{T}\right\rangle}
$$

If $v_{2}\left(p_{T}\right)$ is linearly proportional to $p_{T}$, it is easy to show that Eq. (10) holds exactly. Thus a change of slope in $v_{2}\left(p_{T}\right)$ due to hadronic rescatterings could be attributed to changes of $v_{2}$ and/or $\left\langle p_{T}\right\rangle$, which provides us with more intuitive understanding.

We next calculate $\left\langle p_{T}\right\rangle$ and $p_{T}$-averaged $v_{2}$ for each identified hadron in $|y|<1$ in minimum bias $\mathrm{Au}+\mathrm{Au}$ collisions at $\sqrt{s_{N N}}=200 \mathrm{GeV}$ with the three different options mentioned above. We show $\left\langle p_{T}\right\rangle$ and $v_{2}$ as functions of hadron mass in Figs. 7 (a) and (b), respectively. Default results using the option (I) are shown with closed squares. To see the effect of hadronic rescatterings, we use the option (II) in the hadronic cascade calculation. We also use the option (III) to obtain the results excluding the contributions of both hadronic rescatterings and resonance decays. In the default option (I) in which both hadronic rescatterings and resonance decays are included, $\left\langle p_{T}\right\rangle$ is not a linear function of the hadron mass. By switching off hadronic rescatterings by employing the option (II), we are able to extract the hadronic rescattering effects on evolution of mean $p_{T}$ in the late hadronic stage. The difference of $\left\langle p_{T}\right\rangle$ between with and without hadronic rescatterings are relatively smaller for $\pi, \phi, \Xi$, and $\Omega$ than for the others. In order to confirm a pure flow effect we also calculate $\left\langle p_{T}\right\rangle$ with option (III) in which resonance decay contributions are also not included. As is well-known in analysis using the blast wave model [75], $\left\langle p_{T}\right\rangle$ is a linear function of the hadron mass in this case [76]. We also confirm that it is the case even in our fully dynamical calculations. $p_{T}$-averaged $v_{2}$ for three options (I), (II), and (III) are compared with each other in Fig. 7 (b). By comparing the results (I) and (II) one finds that due to hadronic rescatterings $v_{2}$ for hadrons other than pions increases little - by at most $\sim 6 \%$ - but $v_{2}$ for pions increases by $\sim 25$ even though elliptic flow parameters are commonly believed to be sensitive to the early stage of the reaction. On the other hand, the effect of hadronic rescattering on pion mean $p_{T}$ is almost absent [7]. It is interesting to note that the effect of resonance decays on pion $v_{2}$ is also significant, see Fig. 7 (b). How resonance decays reduce pion $v_{2}$ was discussed in Ref. [77]. As we expected, neither $\left\langle p_{T}\right\rangle$ nor $v_{2}$ for multistrange hadrons is affected by hadronic rescatterings, but at least one of these observables is affected for all other particles.

To see how much hadronic rescatterings affect the final observables, we show ratios of $\left\langle p_{T}\right\rangle$ and $v_{2}$ at particlization but after decays (option (II)) to the ones in the final state (option (I)) 78] for each identified hadron in Fig. 8. For comparison, we also show the ratio obtained from a $m_{T}$ scaling ansatz (open square) [76] in the case of mean $p_{T}$. In this ansatz, one can parametrize $p_{T}$ distribution as

$$
\begin{aligned}
\frac{d N}{p_{T} d p_{T}} & \propto \exp \left(-\frac{m_{T}}{T_{\text {eff }}}\right), \\
T_{\text {eff }} & =T_{f}+\frac{1}{2} m v_{f}^{2}, \\
m_{T} & =\sqrt{p_{T}^{2}+m^{2}},
\end{aligned}
$$

where $T_{f}, m$, and $v_{f}$ are freeze-out temperature, particle mass, and flow velocity, respectively. Parameters were chosen to reproduce the slope of the proton $p_{T}$ spectrum in option (II) $\left(T_{f}=0.155 \mathrm{GeV}\right.$ and $\left.v_{f}=0.56\right)$ and in option (I) $\left(T_{f}=0.130 \mathrm{GeV}\right.$ and $\left.v_{f}=0.71\right)$. If all the particles interact strongly with each other and move at a common collective velocity during the hadronic stage, mass ordering would appear. In fact, the result from the $m_{T}$ scaling depends monotonically on particle mass, and pions, kaons, and protons follow this monotonic tendency obtained from $m_{T}$ scaling ansatz. However, multistrange hadrons (including "hidden" strangeness) $(\phi, \Xi$, and $\Omega$ ) in the integrated dynamical approach deviate from the pattern. This means that multistrange hadrons freeze out earlier than other hadrons and do not participate in the radial flow during the transport stage. Multistrange hadrons couple weakly with the system consisting mostly of pions since they have small scattering cross sections and hardly rescatter with pions in the late hadronic stage.

Mean transverse momentum $\left\langle p_{T}\right\rangle$ for dominant constituents of the medium, namely pions, decreases during the hadronic rescattering stage since $p d V$ work done in the longitudinal direction reduces the transverse energy per unit rapidity [79, 80] but the number of pions is fixed. In this way,

$$
\frac{\left\langle p_{T}\right\rangle_{\text {fluid }_{\text {fl }}}}{\left\langle p_{T}\right\rangle_{\text {final }}} \approx \frac{\left.\left(1 / N_{\pi}\right)\left(d E_{T} / d y\right)_{(\text {fluid }}\right)}{\left(1 / N_{\pi}\right)\left(d E_{T} / d y\right)_{(\text {final })}}>1 .
$$

In fact, the two parameter sets in the $m_{T}$ scaling ansatz mentioned above are chosen to obey this inequality (14). As for $v_{2}$ in Fig. 8 (b), about $20 \%$ of final $v_{2}$ of pions is generated during the late transport stage. In other words, only $\sim 80 \%$ of final $v_{2}$ reflects elliptic flow generated during the fluid-dynamical stage. Obviously, this could depend on centrality (and perhaps collision energy) and the number can be regarded as an average value at the RHIC energy. Whereas hadronic rescatterings affect little final $v_{2}$ for the other hadrons. By combining 

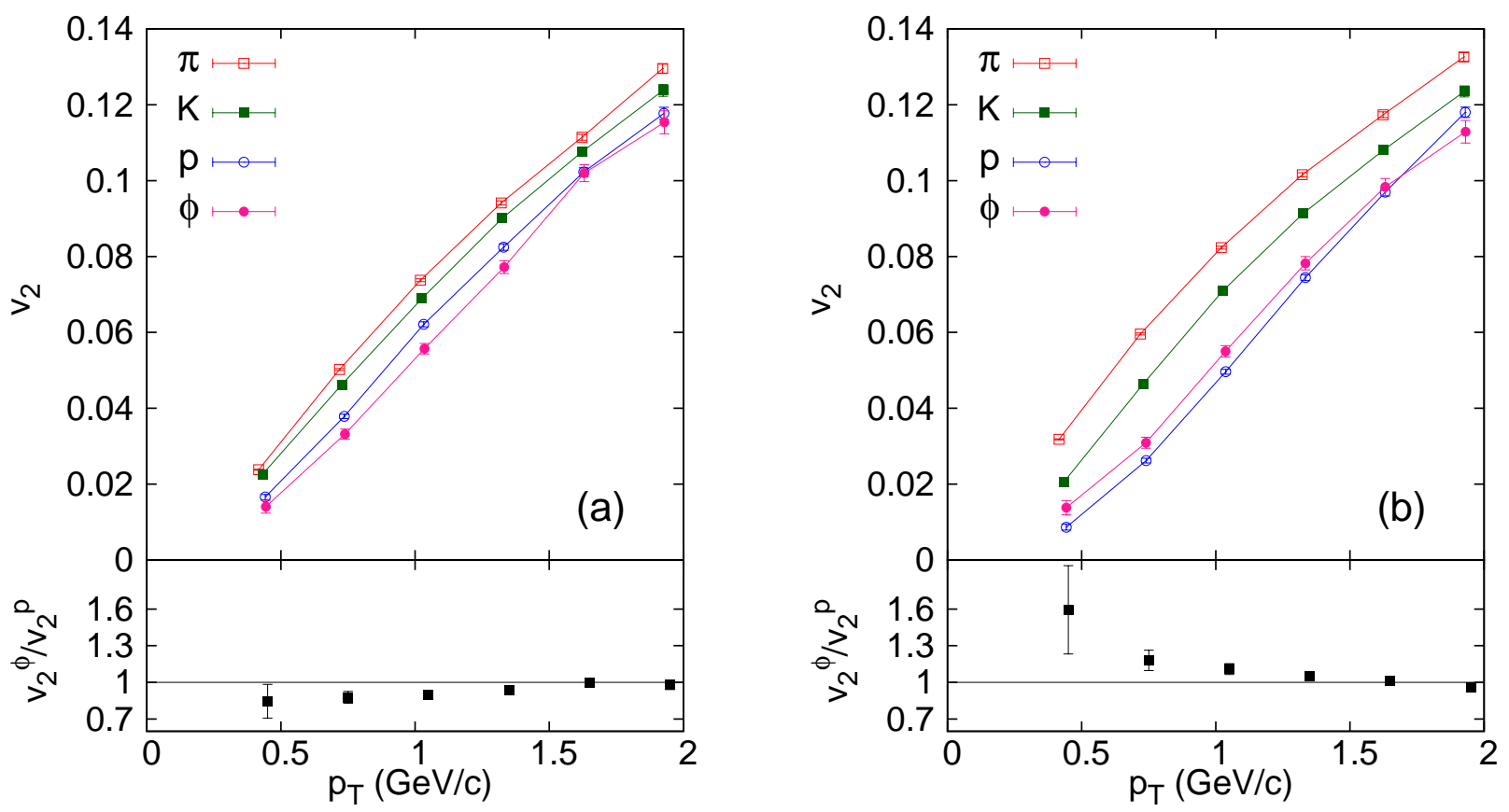

FIG. 6: (Color Online) Transverse momentum dependence of elliptic flow parameter $v_{2}$ for pions (open square), kaons (filled square), protons (open circle), and $\phi$ mesons (filled circle) in $|\eta|<1.0$ obtained from the integrated dynamical approach (a) without hadronic rescattering and (b) with hadronic rescattering in minimum bias $\sqrt{s_{N N}}=200 \mathrm{GeV} \mathrm{Au+Au} \mathrm{collisions}$. The lower panels of the plots show the ratio of $v_{2}^{\phi}$ to $v_{2}^{p}$.
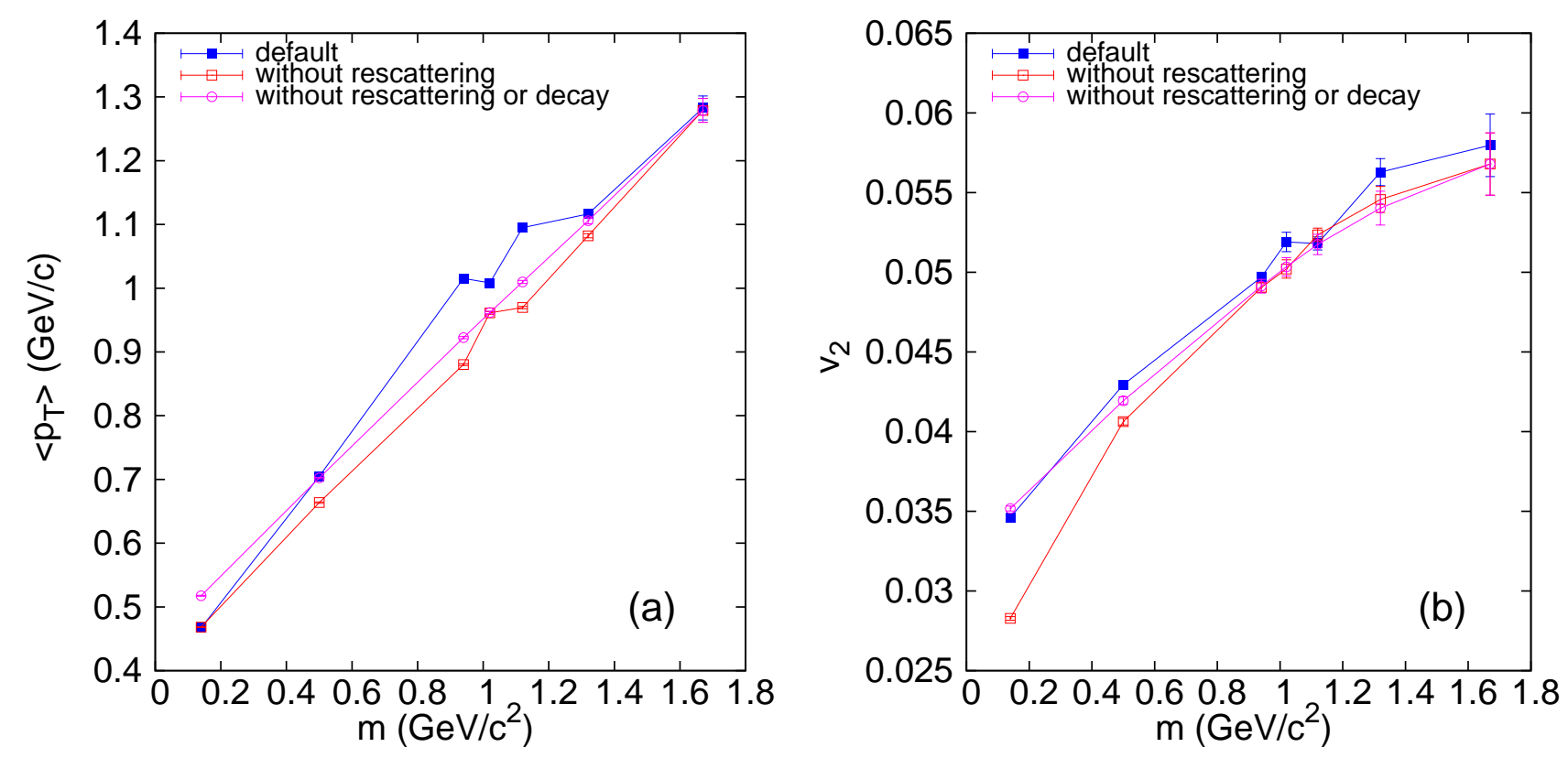

FIG. 7: (Color Online) (a) Mean transverse momentum $\left\langle p_{T}\right\rangle$ and (b) $p_{T}$-averaged $v_{2}$ in minimum bias $\sqrt{s_{N N}}=200 \mathrm{GeV} \mathrm{Au}+\mathrm{Au}$ collisions around midrapidity $(|y|<1.0)$ obtained by the hybrid model as a function of the hadron mass. Each point represents the result for $\pi, K, p, \phi, \Lambda, \Xi$, and $\Omega$ from left to right. Three lines show the results with different options respectively: (I) full calculations with both resonance decays and hadronic rescsatterings (filled square), (II) calculations with resonance decays only (open square), and (III) calculations without resonance decays or hadronic rescatterings (open circle). Lines are to guide the eye. 

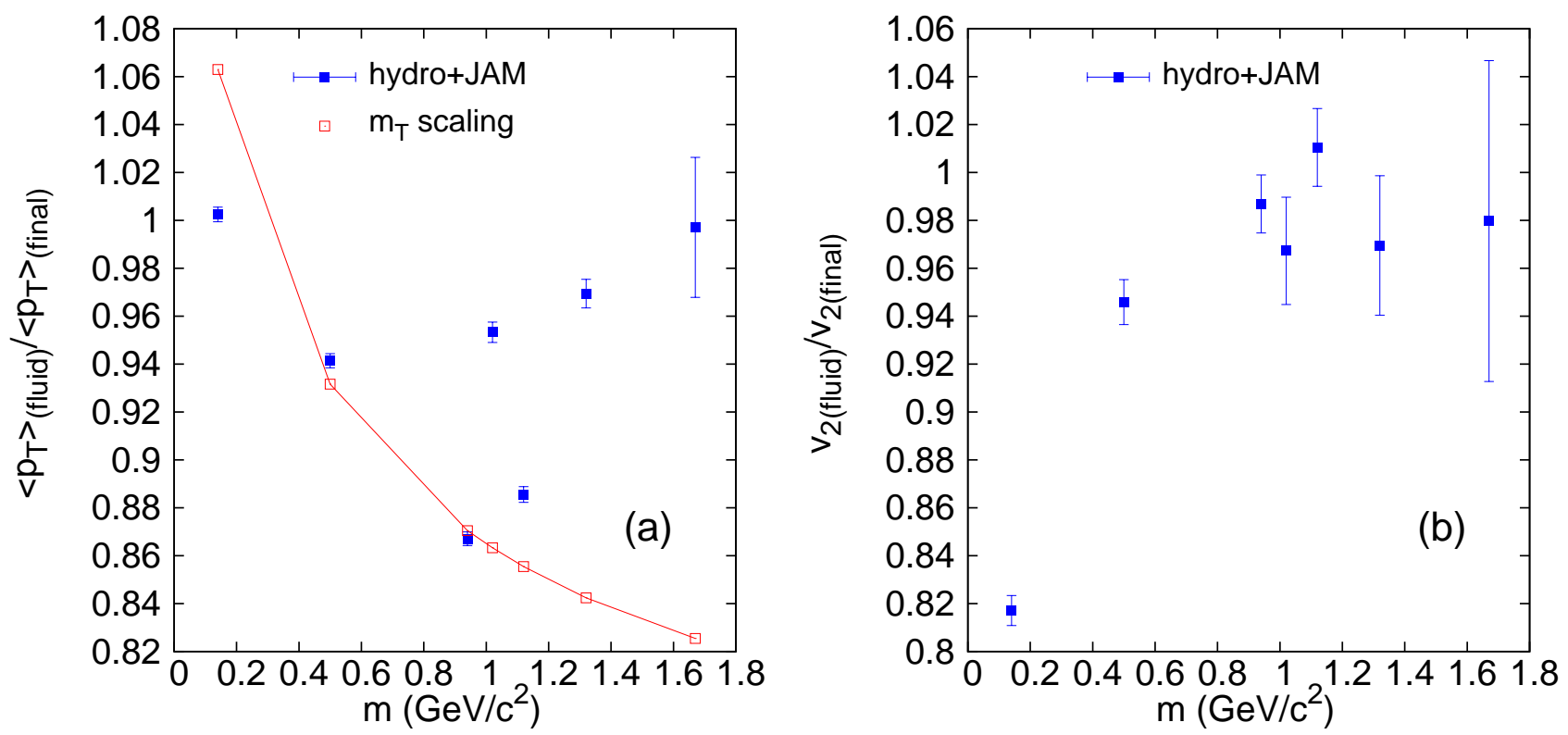

FIG. 8: (Color Online) Ratio of observables at the fluid stage to the final ones in minimum bias $\sqrt{s_{N N}}=200 \mathrm{GeV} \mathrm{Au}+\mathrm{Au}$ collisions in $|y|<1.0$ obtained by the integrated dynamical approach (filled square) for (a) mean transverse momentum $\left\langle p_{T}\right\rangle$ and (b) $p_{T}$-averaged $v_{2}$. In comparison, ratio of $\left\langle p_{T}\right\rangle$ obtained from a transverse mass scaling ansatz (open square) is also shown (see the text). Each point corresponds to a hadron as shown in the caption of Fig. 7

these two results in Fig. 8, we conclude that multistrange hadrons are less affected by hadronic rescatterings and, therefore, can be used to probe the hadronization stage in high-energy nuclear collisions.

Coming back to violation of mass ordering in differential $v_{2}$ shown in Fig. 6 and also in Fig. 5 in Ref. 15, one can interpret this intriguing phenomenon as follows. For pions $p_{T}$-averaged $v_{2}$ increases but $\left\langle p_{T}\right\rangle$ changes little in the hadronic stage, so slope of $v_{2}^{\pi}\left(p_{T}\right)$ gets steeper as seen from Eq. (10). On the other hand, for protons, $p_{T^{-}}$ averaged $v_{2}$ does not change so much, but $\left\langle p_{T}\right\rangle$ increases in the hadronic stage, so $v_{2}^{p}\left(p_{T}\right)$ decreases. However, for $\phi$ mesons, both $p_{T}$-averaged $v_{2}$ and $\left\langle p_{T}\right\rangle$ after the hydrodynamic stage are almost the same in the final state and, consequently, $v_{2}^{\phi}\left(p_{T}\right)$ is unchanged.

Finally we show the normalized freeze-out time (defined as the longitudinal proper time $\tau$ of the last interaction in hadronic cascade simulations) distributions around mid-rapidity $(|y|<1.0)$ in minimum bias $\mathrm{Au}+\mathrm{Au}$ collisions at $\sqrt{s_{N N}}=200 \mathrm{GeV}$. In Fig. 9 the results are shown in two panels, (a) for mesons and (b) for baryons, for clarity. The shaded areas represent the particlization time distribution for charged hadrons. Since the particlization time does not depend on scattering cross sections, it is almost equal for all hadrons within error bars. A prominent peak with small width is seen below $\tau=10 \mathrm{fm} / c$, corresponding to the time of particlization at different parts of the system. The freeze-out time distributions of $\phi$ and $\Omega$ do not differ from the particliza- tion time distribution significantly, which reflects their small scattering cross sections. On the other hand, other hadrons have broader freeze-out time distributions. For $\Xi$, a peak also exists at an early time like for $\phi$ and $\Omega$ but its height is lower and the peak is accompanied by a broad tail. This is because $\Xi$ has a contribution from long-living resonance $\Xi(1530)$ (decay width $\Gamma=9.9 \mathrm{MeV}$ $\sim 1 /(20 \mathrm{fm} / c)$ [4] $)$ whose decay forms a long tail in the freeze-out time distribution. So the primordial $\Xi$ freezes out as early as $\phi$ and $\Omega$ do. These results support our findings above that multistrange hadrons freeze out just after the particlization due to less rescatterings in the late kinetic stage.

\section{SUMMARY}

We have studied the effects of the hadronic rescatterings especially on multistrange hadrons and claimed they can be used to probe the hadronization stage in high-energy nuclear collisions. We have employed an integrated dynamical model in which the ideal hydrodynamic model is combined with the hadronic cascade model, JAM, and confirmed that our model fairly reproduces the experimental data of $p_{T}$ spectra and differential $v_{2}$ not only for pions, kaons, and protons but also for multistrange hadrons. Within this approach, we have simulated the hadronic stage with or without the rescatterings and compared the calculated mean transverse momen- 

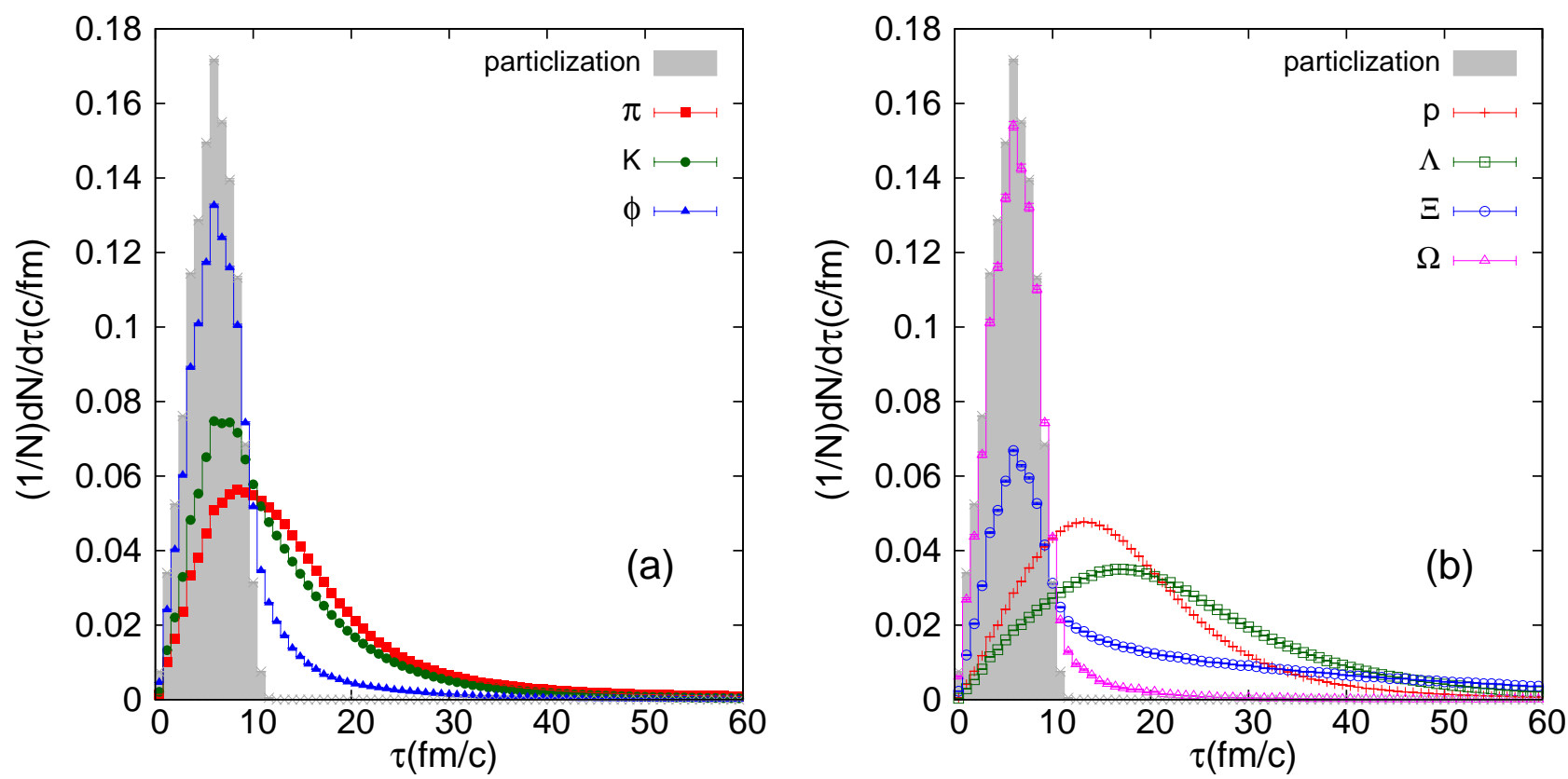

FIG. 9: (Color Online) Normalized freeze-out time $(\tau)$ distributions for (a) mesons: $\pi, K$, and $\phi$, and (b) baryons: $p, \Lambda$, $\Xi$, and $\Omega$ in $|y|<1.0$ in minimum bias $\sqrt{s_{N N}}=200 \mathrm{GeV} \mathrm{Au+Au} \mathrm{collisions.} \mathrm{The} \mathrm{shaded} \mathrm{areas} \mathrm{correspond} \mathrm{to} \mathrm{normalized} \mathrm{particlization}$ time distributions of charged hadrons.

tum and $p_{T}$-averaged $v_{2}$ in these two cases to investigate the hadronic rescattering effects. We have found that the multistrange hadrons are less affected by the rescatterings than non-strange hadrons. Because of the small scattering cross sections, the multistrange hadrons do not fully participate in the radial flow during the hadronic stage and freeze out earlier than non-strange hadrons. With these results we have shown how to interpret behaviors of mass ordering and its violation in $v_{2}\left(p_{T}\right)$. Changes of slope of $v_{2}\left(p_{T}\right)$ during the hadronic rescattering stage result from interplay between changes of mean $p_{T}$ and $p_{T}$-averaged $v_{2}$. We have also showed the freeze-out time distributions for identified hadrons and confirmed that the multistrange hadrons freeze out soon after they are "particlized".

In future, we also plan to investigate the hadronic rescattering effects on observables for multistrange hadrons at the LHC energy. Although elliptic flow parameters are expected to be less affected by the hadronic rescatterings at the LHC, it would be important to quantify how much they do change, and thus how much the observed $v_{2}$ at LHC reflects the QGP evolution.

\section{Acknowledgments}

We acknowledge a fruitful discussion with N. Xu. This work was supported by JSPS KAKENHI Grant Numbers 12J08554(K.M.) and 25400269 (T.H.), and by BMBF under contract no. 06FY9092(P.H.).
[1] K. Yagi, T. Hatsuda, and Y. Miake, Camb. Monogr. Part. Phys. Nucl. Phys. Cosmol. 23, 1 (2005).

[2] U.W. Heinz and P.F. Kolb, Nucl. Phys. A 702, 269 (2002).

[3] M. Gyulassy, arXiv:nucl-th/0403032

[4] T.D. Lee, Nucl. Phys. A 750, 1 (2005).

[5] M. Gyulassy and L. McLerran, Nucl. Phys. A 750, 30 (2005).

[6] E.V. Shuryak, Nucl. Phys. A 750, 64 (2005).

[7] T. Hirano and M. Gyulassy, Nucl. Phys. A 769, 71 (2006).

[8] E.L. Feinberg, Nuovo Cim. A 34, 391 (1976).
[9] E.V. Shuryak, Phys. Lett. B 78, 150 (1978).

[10] E.L. Bratkovskaya, Nucl. Phys. A 931, 194 (2014).

[11] A. Shor, Phys. Rev. Lett. 54, 1122 (1985).

[12] H. van Hecke, H. Sorge, and N. Xu, Phys. Rev. Lett. 81, 5764 (1998).

[13] S.A. Bass and A. Dumitru, Phys. Rev. C 61, 064909 (2000).

[14] Y. Cheng, F. Liu, Z. Liu, K. Schweda, and N. Xu, Phys. Rev. C 68, 034910 (2003).

[15] T. Hirano, U. Heinz, D. Kharzeev, R. Lacey, and Y. Nara, Phys. Rev. C 77, 044909 (2008).

[16] M. He, R.J. Fries, and R. Rapp, Phys. Rev. C 85, 044911 
(2012).

[17] X. Zhu, F. Meng, H. Song, and Y.X. Liu, Phys. Rev. C 91, 034904 (2015).

[18] M. Nasim [STAR Collaboration], Nucl. Phys. A 904-905, 413c (2013).

[19] T. Hirano, P. Huovinen, K. Murase, and Y. Nara, Prog. Part. Nucl. Phys. 70, 108 (2013).

[20] P. Huovinen and P. Petreczky, Nucl. Phys. A 837, 26 (2010).

[21] Y. Nara, N. Otuka, A. Ohnishi, K. Niita, and S. Chiba, Phys. Rev. C 61, 024901 (2000).

[22] A. Bazavov et al., Phys. Rev. Lett. 113, 072001 (2014).

[23] T. Hirano, Phys. Rev. C 65, 011901 (2002).

[24] P. Colella and P.R. Woodward, J. Comput. Phys. 54, 174 (1984).

[25] T. Hirano and Y. Nara, Prog. Theor. Exp. Phys. 2012, 01A203 (2012).

[26] S.A. Bass, A. Dumitru, M. Bleicher, L. Bravina, E. Zabrodin, H. Stoecker, and W. Greiner, Phys. Rev. C 60, 021902 (1999).

[27] D. Teaney, J. Lauret, and E.V. Shuryak, Phys. Rev. Lett. 86, 4783 (2001).

[28] D. Teaney, J. Lauret, and E.V. Shuryak, arXiv:nucl-th/0110037.

[29] T. Hirano, U.W. Heinz, D. Kharzeev, R. Lacey, and Y. Nara, Phys. Lett. B 636, 299 (2006).

[30] C. Nonaka and S.A. Bass, Phys. Rev. C 75, 014902 (2007).

[31] H. Petersen, J. Steinheimer, G. Burau, M. Bleicher and H. Stocker, Phys. Rev. C 78, 044901 (2008).

[32] F. Cooper and G. Frye, Phys. Rev. D 10, 186 (1974).

[33] H. Sorge, A. von Keitz, R. Mattiello, H. Stöcker, and W. Greiner, Z. Phys. C 47, 629 (1990).

[34] H. Sorge, L. Winckelmann, H. Stöcker, and W. Greiner, Z. Phys. C 59, 85 (1993).

[35] S.A. Bass et al., Prog. Part. Nucl. Phys. 41, 225 (1998).

[36] M. Bleicher et al., J. Phys. G 25, 1859 (1999).

[37] C.M. Ko, V. Koch, and G.Q. Li, Ann. Rev. Nucl. Part. Sci. 47, 505 (1997).

[38] X.S. Fang, C.M. Ko, G.Q. Li, and Y.M. Zheng, Nucl. Phys. A 575, 766 (1994).

[39] W. Cassing and E.L. Bratkovskaya, Phys. Rept. 308, 65 (1999).

[40] W. Cassing, Eur. Phys. J. ST 168, 3 (2009).

[41] O. Buss, T. Gaitanos, K. Gallmeister, H. van Hees, M. Kaskulov, O. Lalakulich, A.B. Larionov, T. Leitner, J. Weil, and U. Mosel, Phys. Rept. 512, 1 (2012).

[42] C. Hartnack, H. Oeschler, Y. Leifels, E.L. Bratkovskaya, and J. Aichelin, Phys. Rept. 510, 119 (2012).

[43] R. Rapp and E.V. Shuryak, Phys. Rev. Lett. 86, 2980 (2001).

[44] J. Steinheimer, J. Aichelin, and M. Bleicher, Phys. Rev. Lett. 110, 042501 (2013).

[45] Y. Pan and S. Pratt, Phys. Rev. C 89, 044911 (2014).

[46] P. Huovinen and J.I. Kapusta, Phys. Rev. C 69, 014902 (2004).

[47] K.A. Olive et al. [Particle Data Group], Chin. Phys. C, 38, 090001 (2014).

[48] M.L. Miller, K. Reygers, S.J. Sanders, and P. Steinberg, Ann. Rev. Nucl. Part. Sci. 57, 205 (2007).

[49] H.-J. Drescher and Y. Nara, Phys. Rev. C 75, 034905
(2007).

[50] H.-J. Drescher and Y. Nara, Phys. Rev. C 76, 041903 (2007).

[51] J.D. Bjorken, Phys. Rev. D 27, 140 (1983).

[52] S.S. Adler et al. [PHENIX Collaboration], Phys. Rev. C 69, 034909 (2004).

[53] C. Adler et al. [STAR Collaboration], Phys. Rev. Lett. 89, 202301 (2002).

[54] B.I. Abelev et al. [STAR Collaboration], Phys. Rev. Lett. 97, 152301 (2006).

[55] J. Adams et al. [STAR Collaboration], Phys. Rev. Lett. 92, 112301 (2004).

[56] B.I. Abelev et al. [STAR Collaboration], Phys. Rev. C 79, 064903 (2009).

[57] J. Adams et al. [STAR Collaboration], Phys. Rev. Lett. 98, 062301 (2007).

[58] J. Cleymans, B. Kampfer, M. Kaneta, S. Wheaton, and N. Xu, Phys. Rev. C 71, 054901 (2005).

[59] P. Huovinen, Eur. Phys. J. A 37, 121 (2008).

[60] A. Andronic, P. Braun-Munzinger, and J. Stachel, Phys. Lett. B 673, 142 (2009) [Erratum-ibid. B 678, 516 (2009)].

[61] L. Milano [ALICE Collaboration], Nucl. Phys. A 904905, 531c (2013).

[62] C. Adler et al. [STAR Collaboration], Phys. Rev. Lett. 89, 092301 (2002).

[63] A. Majumder and B. Muller, Phys. Rev. Lett. 105, 252002 (2010).

[64] A. Wiranata, V. Koch, M. Prakash and X.N. Wang, Phys. Rev. C 88, no. 4, 044917 (2013).

[65] R. Hagedorn, Nuovo Cim. Suppl. 3, 147 (1965).

[66] U. Loring, B. C. Metsch and H.R. Petry, Eur. Phys. J. A 10, 395 (2001).

[67] U. Loring, B. C. Metsch and H.R. Petry, Eur. Phys. J. A 10, 447 (2001).

[68] C. Greiner, P. Koch-Steinheimer, F.M. Liu, I.A. Shovkovy and H. Stoecker, J. Phys. G 31, S725 (2005).

[69] J. Noronha-Hostler, J. Noronha, G.S. Denicol, R.P.G. Andrade, F. Grassi and C. Greiner, Phys. Rev. C 89, no. 5, 054904 (2014).

[70] J. Noronha-Hostler and C. Greiner, arXiv:1405.7298 [nucl-th].

[71] J. Adams et al. [STAR Collaboration], Phys. Rev. C 72, 014904 (2005).

[72] T. Hirano and Y. Nara, Phys. Rev. C 79, 064904 (2009).

[73] B.I. Abelev et al. [STAR Collaboration], Phys. Rev. C 77, 054901 (2008).

[74] P. Huovinen, P.F. Kolb, U.W. Heinz, P.V. Ruuskanen, and S.A. Voloshin, Phys. Lett. B 503, 58 (2001).

[75] E. Schnedermann, J. Sollfrank, and U.W. Heinz, Phys. Rev. C 48, 2462 (1993).

[76] H. Heiselberg and A.M. Levy, Phys. Rev. C 59, 2716 (1999).

[77] T. Hirano, Phys. Rev. Lett. 86, 2754 (2001).

[78] T. Hirano, P. Huovinen, and Y. Nara, Phys. Rev. C 83, 021902 (2011).

[79] M. Gyulassy and T. Matsui, Phys. Rev. D 29, 419 (1984).

[80] P.V. Ruuskanen, Phys. Lett. B 147, 465 (1984). 